\title{
Effects of Misalignment of Parallel Beam Optics on Thin Film Stress Analysis
}

\author{
Nicholas Norberg and Arnold C. Vermeulen* \\ PANalytical, Lelyweg 1, 7602 EA Almelo, The Netherlands \\ *arnold.vermeulen@panalytical.com
}

\begin{abstract}
Keywords: X-ray diffraction, residual stress, thin film stress, alignment error, grazing incidence, parallel beam geometry.
\end{abstract}

\begin{abstract}
Collecting reliable data is crucial in the research of residual stresses in thin films using X-ray diffraction. The parallel beam geometry has advantage of reliability compared to focusing beam geometry. Though care must be taken to the alignment. A small alignment error may cause a significant error in the stress value. We will show the sensitivity for the misalignment of the parallel beam optics, discuss requirements on hardware alignment and demonstrate a software correction for the presence of remaining hardware errors.
\end{abstract}

\section{Introduction}

In XRD residual stress analysis it is important to collect reliable data. In general on bulk samples $\mathrm{XRD}$ stress analysis is performed with the single $\{h k l\}$ method and focusing beam optics geometry $[1,2]$. Such a setup needs special attention on the alignment of the incident beam and the alignment of the sample height. This holds for both common variants: the chi stress - side-inclination - mode or the omega stress - iso-inclination - mode $[3,4,5]$. Therefore it is a prerequisite that the chosen instrumental setup is validated first with a measurement on a stress-free powder sample. To reduce the risk for loss of calibration it is essential to repeat the validation measurement with high frequency (e.g. daily).

Employing the parallel beam optics geometry removes the sensitivity for the alignment of the incident beam and sample height [6,7]. This has additional benefits for analyzing curved and rough samples. The primary validation on a stress-free sample can be just considered as a 'formal' step as the test will be easily passed in a normal situation. Due to the intrinsic insensitivity of the parallel beam geometry there is no risk for loss of calibration. Hence, repeat of the validation can be done with lower frequency (e.g. weekly or monthly).

In XRD stress analysis on polycrystalline thin films and coatings the multiple $\{h k l\}$ grazing incidence technique with parallel beam optics geometry is employed. The use of multiple $\{h k l\}$ peaks at different diffraction angles makes the method sensitive for peak position errors on an absolute basis rather than on a relative basis like the single $\{h k l\}$ methods [5,8,9]. Again the parallel beam geometry makes the setup insensitive for most, but not all, errors [6].

In this paper we will look in more detail to the alignment errors relevant for the multiple $\{h k l\}$ grazing incidence technique combined with the parallel beam geometry. The sensitivity for the zero beam error is known [6]. Though it resulted that it is not sufficient to only recalibrate the zero of the $2 \theta$ axis to the measured position of the direct beam.

We demonstrate that a small alignment error may cause a significant error in the residual stress value. Even a change in sign is possible when the absolute stress value is relatively low.

The parallel plate collimator on the diffracted beam side must be aligned properly first. Both angular and spatial aberrations of the parallel plate collimator must be taken into account.

\section{Theory}

In an ideal diffractometer no misalignments are present and perfect measurements can be performed. In practice the ideal diffractometer does not exist and each component has an aberration contributing to the total error in the measurement data. 
Many components of a laboratory X-ray diffractometer are designed to be adjustable in position and/or angle to meet the high demands of the diffraction technique. These parts are adjusted such that the total combined error is reduced. This means that certain errors will be compensated by other errors.

In general instrument aberrations have their influence on the accuracy of the application. The above mentioned error compensation may be desired for one application, but not wanted for another.

For residual stress measurements with the multiple $\{h k l\}$ grazing incidence technique employing the parallel beam geometry the error in the zero $2 \theta$ is the most relevant source for errors in the final stress results. The method of calibration has a different influence on the final result. In the next sections three different cases are presented.

Zero $2 \theta$ calibration with direct beam - incident beam misaligned. A common method to calibrate the zero of the $2 \theta$ axis is using a scan of the direct beam. As shown in Fig. 1 this method results in a propagation of the error in alignment of the incident beam. Although the zero $2 \theta$ seems calibrated and the remaining error is virtually none the alignment procedure has introduced a displacement with respect to the true zero.

An equatorial incident beam misalignment, $\mathrm{h}_{\mathrm{eq}}$, introduces a zero beam error, $\Delta 2 \theta_{\text {zero, }}$, according to

$\Delta 2 \theta_{\text {zero }}\left(^{\circ}\right)=2 \alpha_{\text {inc }}=\frac{180}{\pi} \cdot \frac{2 h_{e q}}{R}$.

This hidden zero beam displacement will affect the residual stress results.

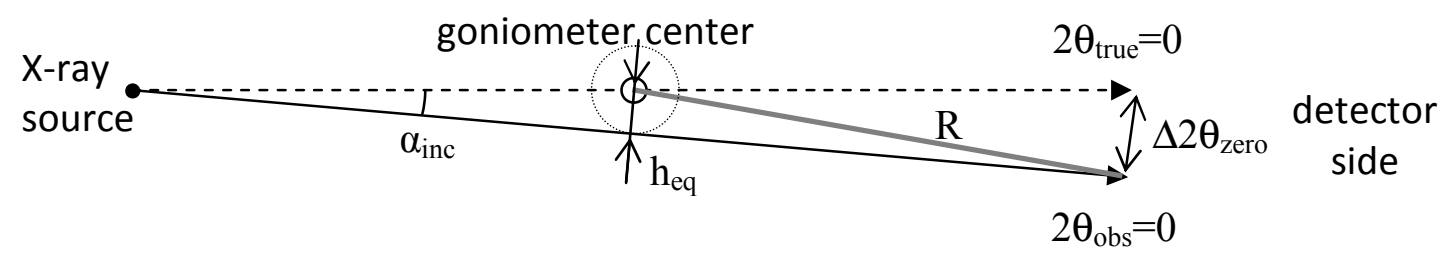

Figure 1. Zero $2 \theta$ calibrated with direct beam with a misaligned incident beam. In the ideal situation the direct beam goes through the goniometer center (dashed line). In practice the incident beam is tilted with angle $\alpha_{\text {inc }}$ and the zero beam is observed at a different angle (solid line). In the calibrated situation then a zero beam displacement is introduced.

Zero $2 \theta$ calibration with direct beam - diffracted beam misaligned. In the parallel beam geometry a parallel plate collimator (PPC) in the diffracted beam is employed. This collimator is sensitive for misalignment of the equatorial tilt, $\alpha_{\mathrm{ppc}}$ or equatorial shift, $\mathrm{h}_{\mathrm{ppc}}$. Ideally the collimator is directed towards the goniometer center. As shown in Fig. 2 both errors lead to an error in the observed position of the zero $2 \theta$.

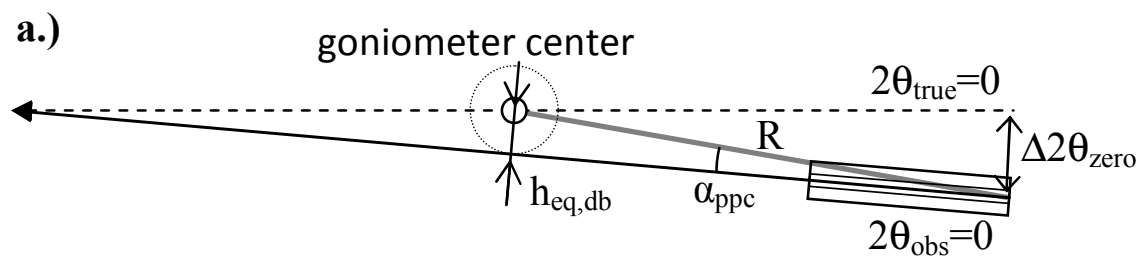

b.)

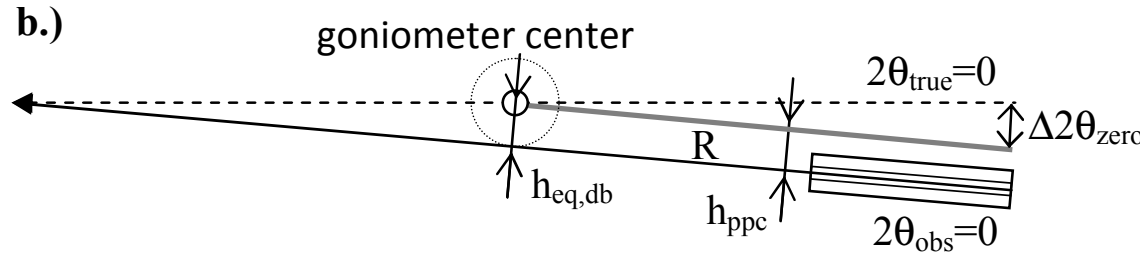

Figure 2. Zero $2 \theta$ calibrated with direct beam using a misaligned parallel plate collimator (PPC) in the diffracted beam. In the ideal situation the observed diffracted beam goes through the goniometer center (dashed line). The PPCis misaligned either with a tilt angle (a.) or a parallel shift (b.) with respect to the diffracted beam arm (grey line). In both cases the direct beam is observed at non-zero angle (solid line) and a zero beam displacement is introduced. 
An equatorial diffracted beam misalignment of the parallel plate collimator, $\mathrm{h}_{\mathrm{eq}, \mathrm{db}}$, introduces a zero beam error, $\Delta 2 \theta_{\text {zero, }}$ according to (see cases (a.) and (b.) in Fig. 2 respectively):

$$
\Delta 2 \theta_{\text {zero }}\left({ }^{\circ}\right)=2 \alpha_{p p c}=\frac{180}{\pi} \cdot \frac{2 h_{e q, d b}}{R}, \quad \Delta 2 \theta_{\text {zero }}\left({ }^{\circ}\right)=\frac{180}{\pi} \cdot \frac{h_{p p c}}{R}=\frac{180}{\pi} \cdot \frac{h_{e q, d b}}{R}
$$

Zero $2 \theta$ calibration with diffraction. An alternative method to calibrate the zero $2 \theta$ is using diffraction measurements on a stress-free powder sample. In this method the total error in the zero $2 \theta$ is determined whether this is caused by a misalignment of the incident beam, diffracted beam or a combination of both. With comparing the peak positions of the complete pattern to their theoretical values the true zero beam position can be found. This value can be used to calibrate the $2 \theta$ scale of the instrument. Alternatively the stress measurement data are corrected for this shift in zero $2 \theta$ in the analysis software [8].

Alignment criterion. The general criterion for proper instrument calibration for residual stress measurements on a stress-free powder sample is that the slope of the $\sin ^{2} \psi$ plot must be close to zero $[10,11]$. More specifically:

$$
\varepsilon(\text { ppm }) \leq 100 \text {. }
$$

For the calibration of the zero beam with diffraction using multiple $\{h k l\}$ peaks this means that

$$
\left|\Delta 2 \theta_{\text {zero }}\right|\left(^{\circ}\right) \leq 0.005
$$

\section{Experimental}

Multiple $\{h k l\}$ grazing incidence stress measurements were conducted on a PANalytical Empyrean multi-purpose diffractometer equipped with a $\mathrm{Cu}$ LFF HR X-ray tube and the PreFIX concept (Pre-aligned Fast Interchangeable X-ray modules) for optics and sample stages. The incident beam path consisted of a parallel beam X-ray mirror. Samples were placed on a sample platform with a programmable $\mathrm{z}$ mechanism. The diffracted beam path consisted of a parallel plate collimator (PPC) with an angular acceptance of $0.27^{\circ}$ in front of a Xe proportional counter.

Alignment of incident and diffracted beam path were extensively checked prior to and after the experiments. Two general alignment states of the same instrument were investigated. In the initial state measurements were conducted on the system before incident and diffracted beam path were aligned, in the aligned state complete alignment incident and diffracted beam path was achieved. In order to investigate the effect of the PPC on residual stress measurements controlled tilt misalignment of the PPC was created by clamping $\mathrm{Cu}$-foils with different thicknesses (50 and 100 $\mu \mathrm{m}$ ) in positions 1 and 2 between the T- and the U-block of the PreFIX (Fig. 3).

The influence of the induced PPC misalignment was determined via scans through the direct beam with a PPC as well as measurements of a stress-free W powder sample. From the direct beam scans the zero $2 \theta$ shift was directly measured by determining the center of gravity of intensity from the respective profiles (Fig. $4 \mathrm{a}$ ). On the other hand $\mathrm{W}$ powder scans were used to calculate and calibrate the zero $2 \theta$ shift by performing multiple $\{h k l\}$ misalignment analysis (Fig. $4 \mathrm{~b}$ ). In addition a TiN coating on steel with a known residual stress was measured with the aligned and misaligned setup to test whether the determined zero $2 \theta$ calibration is able to correct for the respective errors. Both, misalignment analysis as well as misalignment correction were conducted using the PANalytical Stress Plus 2.1 software package. 

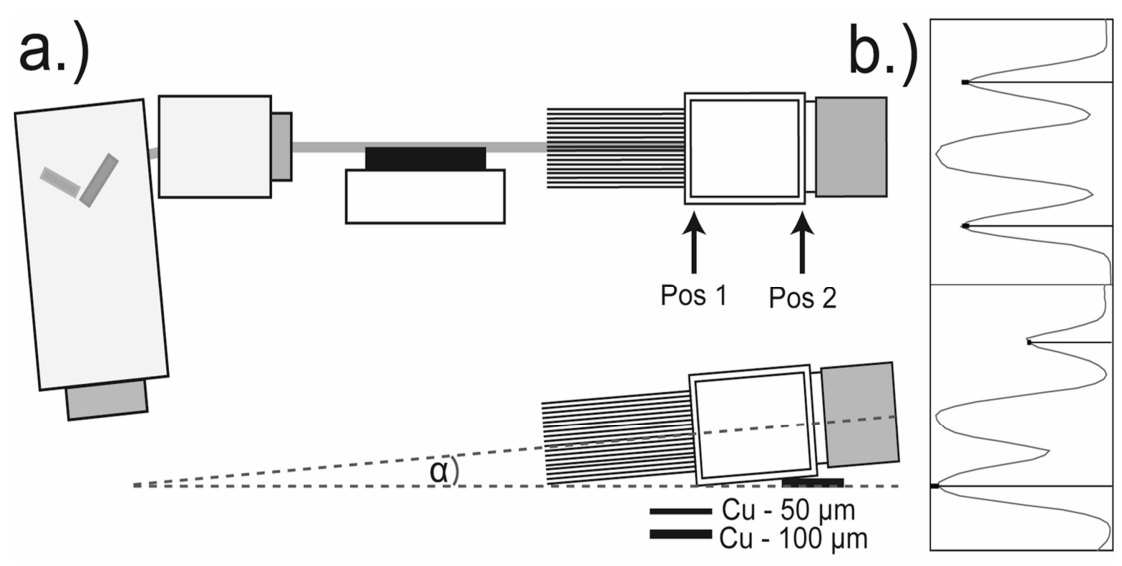

Figure 3. a.) Sketch of the beam path in the aligned case (top) and with a tilt misalignment of the PPC. b.) Profiles showing the effect of the PPC tilt on the detected intensity distribution of the direct beam. The upper profile shows the desired symmetric peak triplet of an aligned PPC, the lower profile demonstrates the effect of a tilt misalignment $(100 \mu \mathrm{m} \mathrm{Cu}$-foil, position 2).
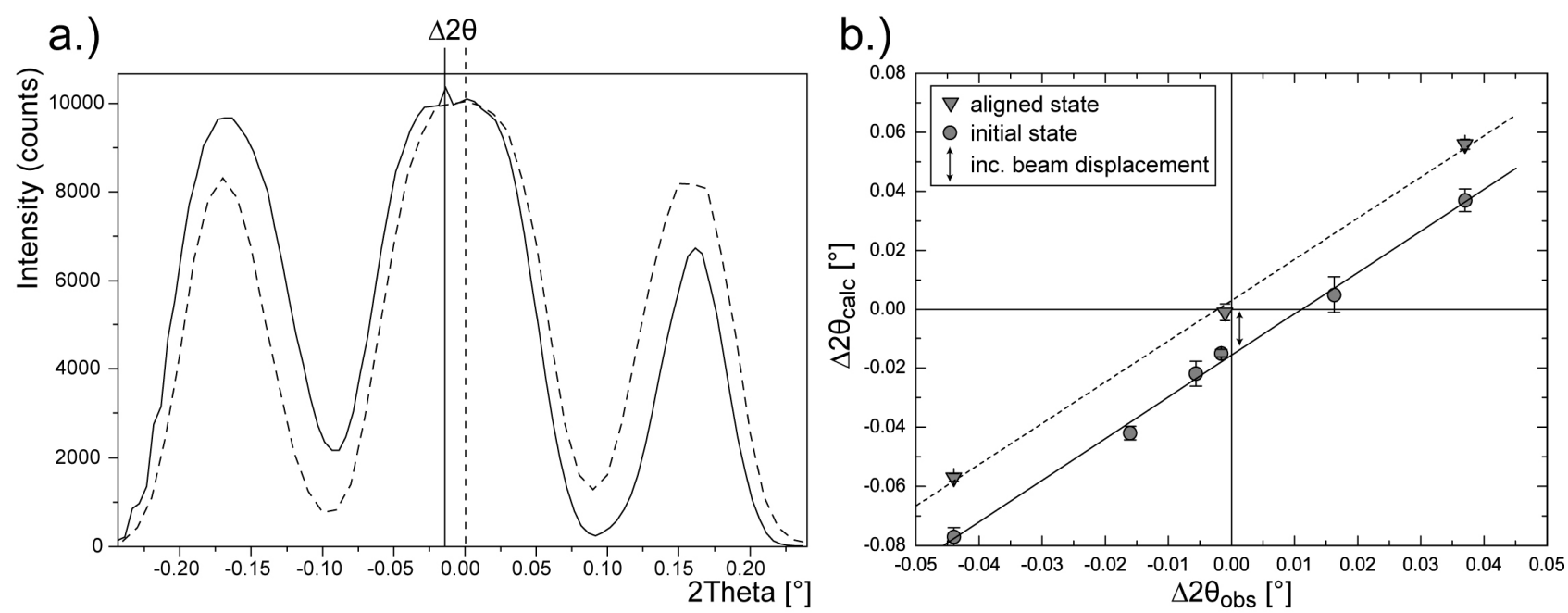

Figure 4. a.) Direct beam profiles of an aligned PPC (dashed line) and a misaligned PPC (50 $\mu \mathrm{m}$ Cu-foil, position 2). Vertical lines indicate the direct beam position defined by the center of gravity of the total profile intensity. b.) Plot showing preliminary results of the observed zero $2 \theta$ shift $\left(\Delta 2 \theta_{\text {obs }}\right)$ determined from the direct beam profile plotted against the zero $2 \theta$ shift calculated from the misalignment analysis $\left(\Delta 2 \theta_{\text {obs }}\right)$ obtained in this study. Data sets obtained with the two different alignment states of the instrument follow the same trend. The vertical shift of the initial state data result from an incident beam displacement of $0.018^{\circ} 2 \theta$ present prior to the alignment of the system.

\section{Results and discussion}

The induced tilt misalignment in the PPC results in a significant $2 \theta$ shift which can be clearly seen from a shift in the intensity profile across the direct beam (Fig. 4a). This shift directly translates into an absolute zero $2 \theta$ misalignment error up to several hundredths of a degree over the whole $2 \theta$ range (Fig. $4 \mathrm{~b}$ ). This introduces a false peak shift unrelated to strain induced by residual stress within the analyzed compound and hence corrupt the slope in an $\varepsilon$ vs. $\sin ^{2} \psi$ plot resulting in a wrong stress value (Fig. 5). In this study we could introduce an additional strain of $\pm 1400 \mathrm{ppm}$ only resulting from a tilt misalignment of the used PPC.

Depending on the elastic properties of the analyzed material this factor alone can result in absolute stress values of several hundreds MPa (Fig. 6). In order to assess the influence on a real multiple $\{h k l\}$ grazing incidence stress analysis, a TiN coating on steel was measured with a completely aligned instrument as well as two misaligned PPC scenarios. Depending on the applied PPC tilt misalignment, deviations of $-657 \mathrm{MPa}$ and $+497 \mathrm{MPa}$ from the real value of $-10699 \mathrm{MPa}$ could be created. These values seem to be relatively insignificant when dealing with total stress values in the range of $10 \mathrm{GPa}$. However, since our results clearly indicate that this error is absolute rather than relative (Fig. 6), it could even result in a change in sign when the expected stress lies in the range of 
a.)

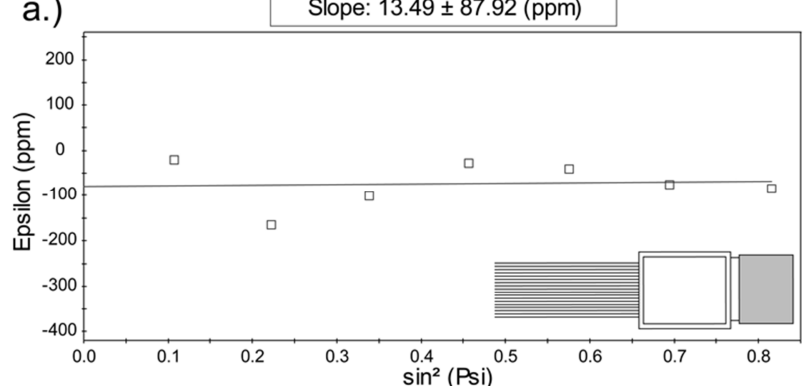

C.)

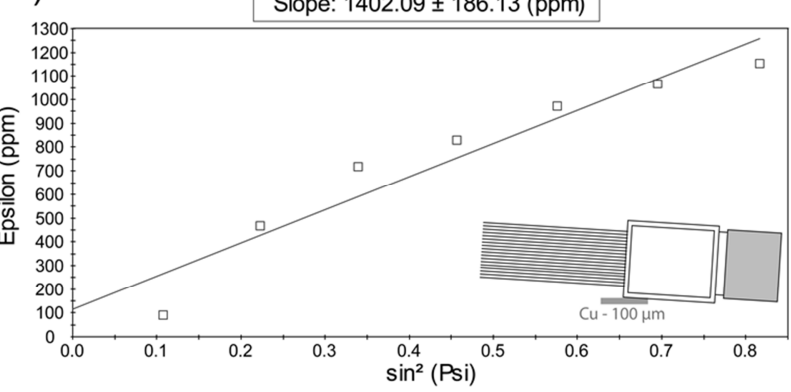

b.)

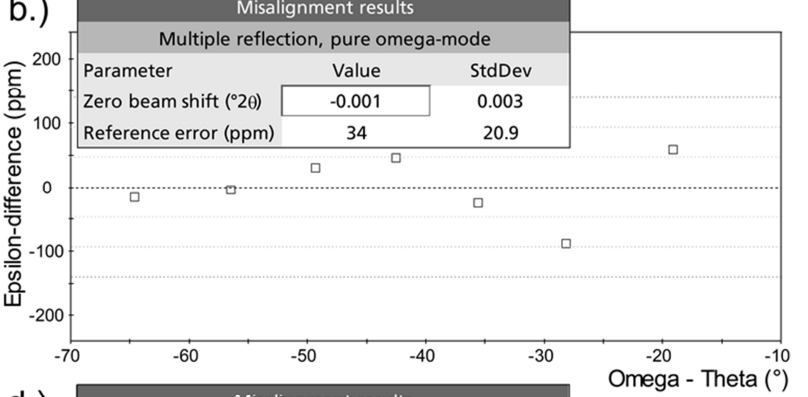

d.)

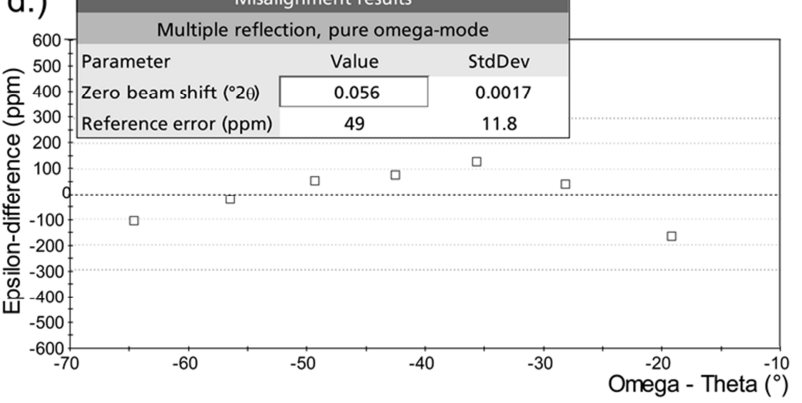

Figure 5. Results of the residual stress analysis (a.) and misalignment analysis (b.) of the stress-free W powder sample in the fully aligned case. The obtained data shows indeed no residual stress as well as no significant zero $2 \theta$ shift. In contrast residual stress (c.) and misalignment analysis (d.) in a case where PPC misalignment was induced by clamping a $100 \mu \mathrm{m}$ thick $\mathrm{Cu}$-foil in position 1 , shows the significant effect on a multiple $\{h k l\}$ grazing incidence stress measurements solely resulting from a misaligned PPC.
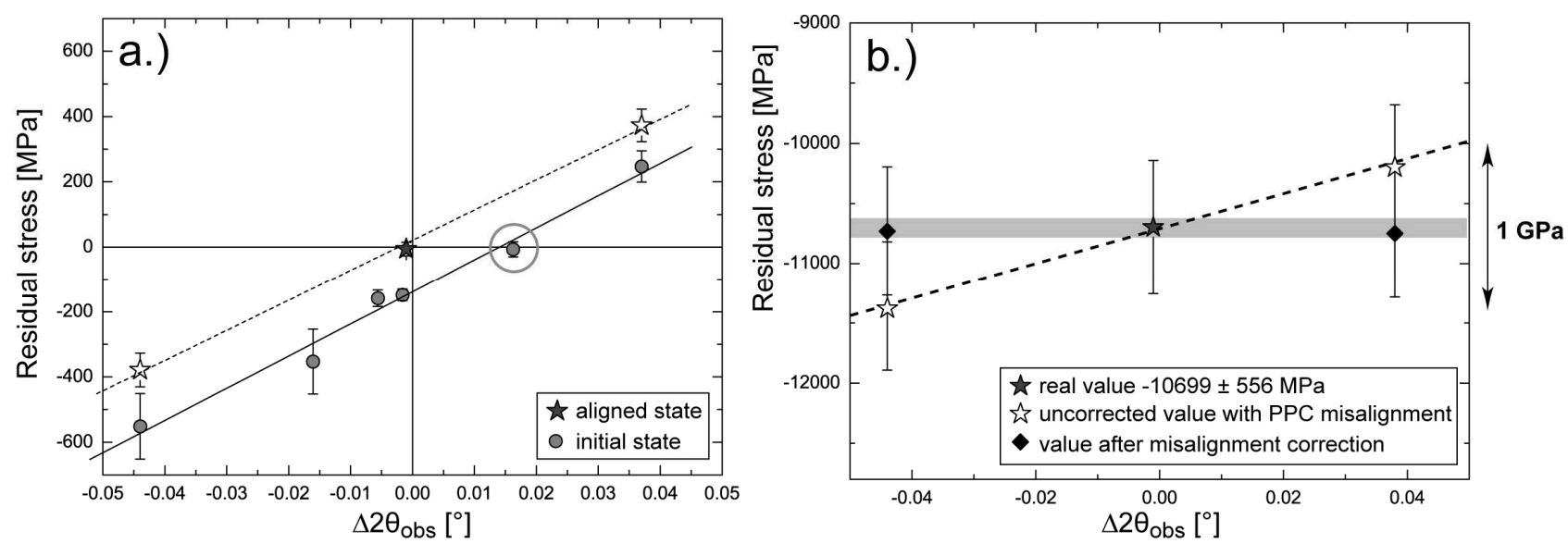

Figure 6. a.) Residual stress data calculated from measurements of the stress-free $\mathrm{W}$ powder sample using isotropic elastic constants of TiN $\left(\mathrm{S}_{1}=-0.44 \mathrm{TPa}^{-1}, 1 / 2 \mathrm{~S}_{2}=2.77 \mathrm{TPa}^{-1}\right)$. A total deviation in the stress value of $\pm 400 \mathrm{MPa}$ could solely be induced by misaligning the PPC. The highlighted data point indicates a scenario where incident and diffracted beam misalignments compensate each other. b.) Stress analysis conducted on a TiN coating on steel using an aligned setup and a setup with a misaligned PPC (100 $\mu \mathrm{m}$ foil on position 1 and 2) before and after conducting a misalignment correction.

0 to $500 \mathrm{MPa}$. Therefore any disregard for the alignment state of the PPC even in cases where the incident beam path is perfectly aligned can completely corrupt the final result.

Any additional misalignment in the optical beam path, e.g. incident beam misalignment, will cause an additional error that can either enhance or reduce the present diffracted beam error. This can be seen in Fig. 6a where data obtained from the stress-free W sample was recalculated using TiN elastic constants. It shows that despite an incident beam misalignment error of $0.018{ }^{\circ} 2 \theta$ the correct stress-free case could be achieved as a result of the presence of the same error in the diffracted beam path. 
Despite the use of a parallel beam setup, in order to remove any influence caused by misalignment on multiple $\{h k l\}$ grazing incidence stress analyses, the use of a stress-free sample in combination with a subsequent misalignment analysis and misalignment correction is unavoidable. As the results shown in Fig. $6 \mathrm{~b}$ indicate, any misalignment error can be corrected to obtain the correct stress value. For the TiN sample, after the misalignment correction the final values of stress were -10730 and $-10748 \mathrm{MPa}$, respectively, which are fairly close to the real value of $-10699 \mathrm{MPa}$.

\section{Conclusions}

The results obtained in this study demonstrate the significant influence of the alignment condition of the PPC on residual stress analyses of thin films or coatings using the multiple $\{h k l\}$ grazing incidence approach. Parallel beam optics remove the sensitivity for errors in the alignment of the incident beam and sample height. However, alignment errors in the parallel plate collimator itself directly translate in a zero $2 \theta$ shift that has to be correctly determined and corrected for prior and during the analysis of an unknown sample. Disregard of this error can lead to significant errors up to several hundreds MPa depending of the magnitude of the PPC tilt. For samples with relatively low residual stresses this can even result in a change of sign (from compressive to tensile and vice versa).

This study conclusively showed that the calibration of the zero $2 \theta$ shift via diffraction using a stress-free powder sample (in this study W powder) is the most reliable approach. It is demonstrated that if a misalignment correction based on the determined zero $2 \theta$ value is applied, reliable stress results are obtained as shown in the example of a TiN coating. Zero $2 \theta$ calibration using a simple direct beam measurement is not sufficient as it does not show the whole extent of the misalignment error.

\section{References}

[1] I.C. Noyan, J.B. Cohen, Residual Stress, Springer-Verlag, New York, 1987.

[2] V.M. Hauk, E. Macherauch, A Useful Guide for X-Ray Stress Evaluation (XSE), Advances in X-ray analysis, 27 (1983) 81-99.

[3] F. Convert, B. Miege, The Control of Geometrical Sources of Error in X-Ray Diffraction Applied to Stress Analysis, J. Appl. Cryst. 25 (1992) 384-390.

[4] A.C. Vermeulen, E. Houtman, Determination of Alignment Errors in Classical XRD Residual Stress Methods. Measurement, Accuracy and Correction, Mat. Science Forum, 347-349 (2000) 17-22.

[5] A.C. Vermeulen, Determination of Alignment Errors in Classical XRD Residual Stress methods. Applicability of a Software Correction, Proc. ICRS-6, Oxford, UK (2000) 283-290.

[6] A.C. Vermeulen, The Sensitivity of Focusing, Parallel Beam and Mixed Optics to Alignment Errors in XRD Residual Stress Measurements, Mat. Science Forum, 490-491 (2005) 131-136.

[7] T. Hanabusa, Japanese Standard for X-ray Stress Measurement, Proc. ICRS-6, Oxford, UK (2000) 181-188.

[8] A.C. Vermeulen, Accurate Absolute Peak Positions for Multiple $\{h k l\}$ Residual Stress Analysis by Means of Misalignment Corrections, Z. Kristallogr., Suppl. 23 (2006) 49-54.

[9] A.C. Vermeulen, Instrumental Aberrations in a 4-Circle Powder Diffractometer, Z. Kristallogr., 222 (2007) 204-209.

[10] EN 15305:2008, Non-destructive Testing - Test Method for Residual Stress Analysis by X-ray Diffraction, European Standard.

[11]A.C. Vermeulen, Considerations for Collecting Reliable Residual Stress Data Across the Full 2Theta Range, Adv. in X-Ray Analysis 49 (2006) 133-142. 